\title{
Neurocysticercosis presenting as isolated wall-eyed monocular internuclear ophthalmoplegia with contraversive ocular tilt reaction
}

\author{
Suresh R Chandran, Rojith K Balakrishnan, K Umakanthan, K Govindarajan ${ }^{1}$ \\ Departments of Medicine and ${ }^{1}$ Neurology, Coimbatore Medical College, Coimbatore, India
}

\section{ABSTRACT}

Neurocysticercosis is a common tropical infection presenting with neurological signs. It commonly presents as seizures but various other focal neurological presentations have been reported. Though neurocysticercosis have been reported to present as isolated internuclear ophthalmoplegia, we report the first case of neurocysticercosis presenting as walleyed monoocular internuclear ophthalmoplegia syndrome with contraversive ocular tilt reaction.

Key words: Internuclear ophthalmoplegia, neurocysticercosis, ocular tilt reaction, wall-eyed bilateral internuclear ophthalmoplegia, wall-eyed monocular internuclear ophthalmoplegia

\section{Introduction}

Internuclear ophthalmoplegia (INO) results from a disruption of the medial longitudinal fasciculus (MLF). This leads to loss of adduction in the ipsilateral eye and dissociated nystagmus on abduction in the contralateral eye. INO can be associated with other neurological signs like skew deviation and ocular tilt reaction (OTR). Neurocysticercosis is a common cause of focal neurological lesions in the tropics. Varied presentations of neurocysticerci have been reported from different parts of the world. We report the first case of INO presenting as wall-eyed monocular internuclear ophthalmoplegia (WEMINO) syndrome with contraversive OTR due to pontine neurocysticercosis.

\section{Case Report}

A 35-year-old man presented with sudden onset of

\begin{tabular}{|l|l|}
\hline \multicolumn{2}{|c|}{ Access this article online } \\
\hline Quick Response Code: & Website: \\
\hline & www.ruralneuropractice.com \\
\cline { 2 - 2 } & \\
\hline & \\
\hline
\end{tabular}

headache, nausea, and giddiness of two-day duration. He did not have any significant medical history. On examination, his vital signs were normal. Neurological examination revealed exotropia of his right eye with hypotropia of his left eye in primary gaze [Figure 1]. His head position was tilted to left resulting in his left eye to be on a lower plane than the right. On attempted left gaze, there was loss of adduction in his right eye and mild dissociated nystagmus in his left eye. On attempted right gaze, his eye movements were normal [Figure 2] [Video 1]. His pupils were equal in size and reacted normally to light. His convergence was impaired on the right. Optokinetic test revealed an adduction lag in his right eye. His vertical saccades were normal. Ocular torsion (OT) and skew deviation were not measured objectively. There was no history of diplopia. Rest of his neurological examinations including fundus and other cranial nerves were normal. The above clinical features are characteristic of INO plus syndrome. MRI brain of the patient showed a typical well-defined cystic lesion with intracavitary scolex on the right side of mid pons [Figure 3]. The evidence of a typical scolex on a CT or MRI is considered the absolute criteria for diagnosis of neurocysticercosis. ${ }^{[1]}$ The presence of unilateral INO with ipsilesional exotropia in primary gaze is characteristic of WEMINO syndrome. The head tilt and skew torsion away from the lesion side comprised

Video available at www.ruralneuropractice.com

Address for correspondence:

Dr. Suresh R. Chandran, Pulari, By Pass Road, PO Shoranur, Kerala - 679121, India. E-mail: drsureshrc@yahoo.com 


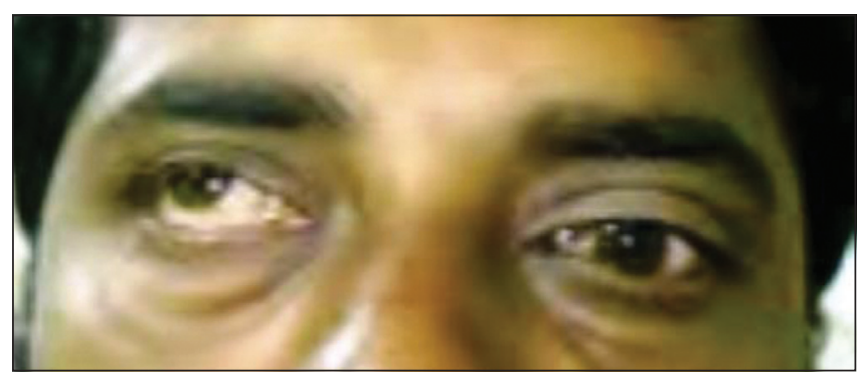

Figure 1: Primary gaze showing right exotropia and left hypotropia with head tilt to left
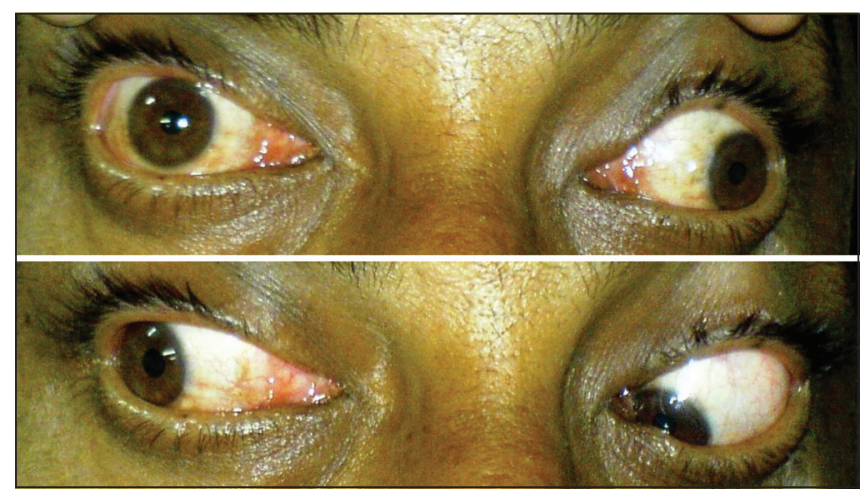

Figure 2: Absent adduction in right eye on attempted left gaze and normal movement on right gaze

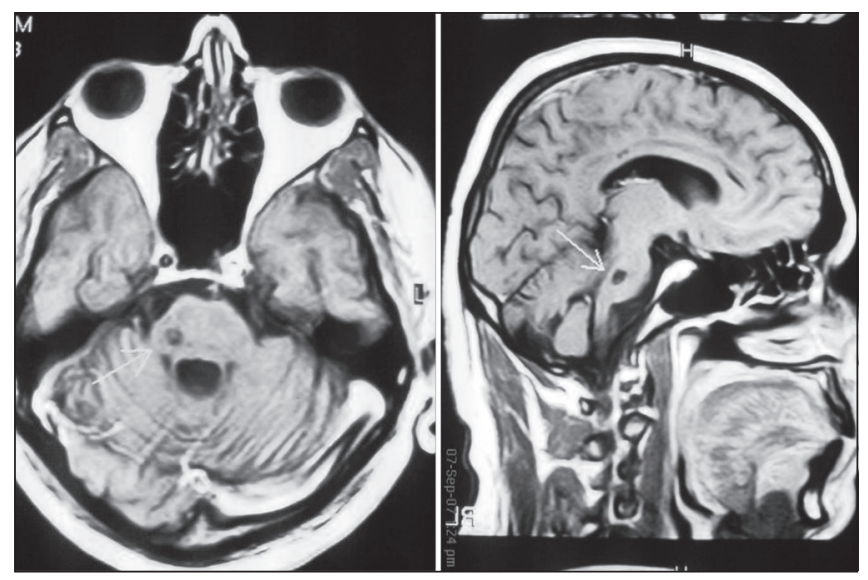

Figure 3: MRI showing the typical well-defined cystic lesion in pons with intracavitary scolex

a contraversive OTR. Thus, this patient has isolated WEMINO syndrome with contraversive OTR.

The patient was started on a course of albendazole and oral steroids. The exotropia and nystagmus disappeared in two days. Patient was discharged after a week with near complete recovery of ocular movements. Follow up after two weeks of onset of symptoms revealed complete recovery of ocular movements.

\section{Discussion}

Neurocysticercosis commonly presents with seizures, or is asymptomatic. But neurocysticercosis has long been recognized as a cause of pretectal or dorsal midbrain syndromes, cerebrovascular complications, and INO ${ }^{[2]}$ Many rarer presentations of neurocysticercosis have been reported. These include vertical diplopia, nystagmus, one and a half syndrome, isolated facial myokymia, bilateral INO, and oculomotor nerve palsy. ${ }^{[3-6]}$ Thus, depending on the location of the lesion, neurocysticerci can have varied manifestations. Other well-known causes of INO include brainstem neoplastic lesions, myasthenia gravis, ArnoldChiari malformation, nutritional and metabolic encephalopathies.

INO can be associated with various additional neurological signs like skew deviation, OT, and OTR. Isolated INO patients have parallel visual axes in primary gaze. When the primary gaze shows exotropia in the ipsilesional eye, it is called WEMINO. ${ }^{[7]}$ If exotropia is contralesional, it is called nonparalytic pontine exotropia (NPPE) ${ }_{1}^{[8]}$ to differentiate it from paralytic pontine exotropia seen in one and a half syndrome. NPPE results from over action of the contralateral Para pontine reticular formation (PPRF) under fixation with the paretic eye. If exotropia is present in both eyes in primary gaze, it is called WEBINO. ${ }^{[9,10]}$

WEBINO is a rare variation of INO associated with lesions of the MLF bilaterally. ${ }^{[11]}$ The distinguishing features of WEBINO are adduction deficits on lateral gaze to both sides and exotropia in primary gaze position. The term is attributed to Lubow according to Hoyt and Daroff in 1971. ${ }^{[12]}$ McGettrick and Eustace have suggested that an oculomotor nucleus lesion accounts for the exotropia in patients with WEBINO, but Gonyea reported that the oculomotor nucleus was not involved. ${ }^{[11,13]}$ Exotropia can also be associated with unilateral INO. Abnormality in the vestibular signals reaching the medial rectus nuclei and resulting disparity in the muscular tone has been postulated to be the cause of exotropia. ${ }^{[14,15]}$ Similarly, an outward deviation in the contralesional eye can result from an imbalance of PPRF signals. ${ }^{[16]}$ Another contradiction to the concept of medial rectus subnucleus involvement in WEBINO has come from the observations that exotropia also occurs ipsilaterally in unilateral INO, that is, in the WEMINO syndrome. Among four patients with WEMINO described by Johnston and Sharpe, pathologic analyses obtained for one patient showed that the lesion was confined to the pontine tegmentum..$^{[7,17]}$ 
OTR comprises a triad of skew deviation, OT, and head tilt. Either complete OTR or skew torsion without head tilt indicates a unilateral deficit of otolith input or a unilateral lesion in the graviceptive pathways from the vestibular nuclei to the interstitial nucleus of Cajal (INC). Rapoport et al. have also reported graviceptive dysfunction in the MLF and surrounding structures to be the cause of body tilt in INO and one and a half syndrome. ${ }^{[18]}$ The pathway crosses at the lower pontine level where the vestibular nuclei are localized. The OTR is ipsiversive if lesion occurs below the decussation, as in peripheral or pontomedullary lesions indicating the involvement of the medial and/or superior vestibular nuclei. OTR is contraversive if lesion occurs above the decussation as in unilateral pontomesencephalic brainstem lesions above the decussation, indicating the involvement of the MLF or INC and rostral interstitial nucleus of MLF. ${ }^{[19]}$ The ipsilesional eye is on a higher plane, with head tilt and OT away from the side of lesion in contraversive OTR. The ipsilesional eye is on a lower plane with head tilt and OT toward the side of lesion in ipsiversive OTR.

In our patient, the lesion was located in mid pons affecting the MLF and the vestibular pathways above the level of decussation, resulting in WEMINO with contraversive OTR. Similar cases of WEMINO and WEMINO with contraversive OTR have been reported in literature. ${ }^{[20,21]}$ But to the best of our knowledge, this case represents the first case of WEMINO syndrome with contraversive OTR caused by neurocysticercosis.

\section{References}

1. Garg RK. Diagnostic criteria for neurocysticercosis: Some modifications are needed for Indian patients. Neurol India 2004;52:171-7.

2. Jha S, Kumar V. Neurocysticercosis presenting as stroke. Neurol India 2000;48:391-4.

3. Jethani J, Vijayalakshmi P, Kumar M. Atypical ophthalmological presentation of neurocysticercosis in two children. J AAPOS 2007;11:495-6.

4. Bhattacharya AK, Mitra K. Bilateral internuclear ophthalmoplegia an atypical presentation of neurocysticercosis. JIACM 2006;7:64-7.

5. Chotmongkol V, Sawanyawisuth K, Limpawattana P, Phuphatham
A, Chotmongkol R, Intapan PM. Superior divisional oculomotor nerve palsy caused by midbrain neurocysticercosis. Parasitol Int 2006;55:223-5.

6. Keane JR. Internuclear ophthalmoplegia unusual causes in 114 of 410 patients. Arch Neurol 2005;62:714-7.

7. Johnston JL, Sharpe JA. The WEMINO syndrome-wall-eyed monocular internuclear ophthalmoplegia: An oculographic and neuropathologic characterization. Neurology 1994;44:311.

8. Komiyama A, Takamatsu K, Johkura K, Hasegawa O, Fukutake T, Hirayama K. Internuclear ophthalmoplegia and contralateral exotropia: Nonparalytic pontine exotropia and WEBINO syndrome. Neuroophthalmology 1998;19:33-44.

9. Jung DS, Park KP. Posttraumatic bilateral internuclear ophthalmoplegia with exotropia. Arch Neurol 2004;61:429.

10. Strominger MB, Mincy EJ, Strominger NL. Bilateral internuclear ophthalmoplegia with absence of convergent eye movements Clinicopathologic correlation. J Clin Neuroophthalmol 1986;6:57-65.

11. McGettrick P, Eustace P. The w.e.b.i.n.o. syndrome. Neuroophthalmology 1985;5:109-15.

12. Hoyt WF, Daroff RB. Supranuclear disorders of ocular control systems in man: Clinical, anatomical, and physiological correlations. In: BachY-Rita P, Colins CC, editors. The Control of Eye Movements. New York: Academic Press; 1971. p. 223-6.

13. Gonyea EF. Bilateral internuclear ophthalmoplegia. Association with occlusive cerebrovascular disease. Arch Neurol 1974;31:168-73.

14. Zhao C, Lu S, Tajouri N, Aliferis K, Landis T, Safran AB. Room tilt illusion influenced by head position. J Neuro-Ophthalmol 2007; 4:297-9.

15. Flitcroft DI, Saidlar CA, Stack JP, Eustace P, A proposed neuroanatomical and neurophysiological basis for WEBINO. Neuroophthalmology 1996;16:280.

16. Johkura K, Komiyama A, Kuroiwa Y. Eye deviation in patients with one-and-a-half syndrome. Eur Neurol 2000;44:210-5.

17. Chen CM, Lin SH. Wall-Eyed bilateral internuclear ophthalmoplegia from lesions at different levels in the Brainstem. J Neuro-Ophthalmol 2007;27:9-15.

18. Rapoport A, Gilad R, Eilam A, Lampl Y, Dabby R, Sadeh M. Dynamic body tilt in internuclear ophthalmoplegia and one-and-a-half syndrome. J Neuro-Ophthalmol 2004;28:137-45.

19. Chen CM, Lin SH. Ipsiversive partial ocular tilt reaction in a patient with acute paramedian thalamic infarctions. Acta neurol taiwan 2006;15:29-33.

20. Ikeda Y, Okamoto K. Lesion responsible for WEMINO syndrome confirmed by magnetic resonance imaging. J Neurol Neurosurg Psychiatry 2002;73:204-5.

21. Jeon SB, Chung SJ, Ahn H, Lee JH, Jung JM, Lee MC. Walleyed monocular internuclear ophthalmoplegia (WEMINO) with contraversive ocular tilt reaction. J Clin Neurol 2005;1:101-3.

How to cite this article: Chandran SR, Balakrishnan RK, Umakanthan K, Govindarajan K. Neurocysticercosis presenting as isolated wall-eyed monocular internuclear ophthalmoplegia with contraversive ocular tilt reaction. J Neurosci Rural Pract 2012;3:84-6.

Source of Support: Nil. Conflict of Interest: None declared. 